\title{
Conscience et connaissance de l'hépatite $C$ chez les fournisseurs de soins de santé et la population en général : examen de la portée
}

\author{
$\mathrm{S} \mathrm{Ha}{ }^{1 *}, \mathrm{~K}$ Timmerman ${ }^{1}$
}

\section{Résumé}

Contexte : La Stratégie mondiale du secteur de la santé vise à éliminer d'ici 2030 la menace à la santé publique qu'est I'hépatite. Le virus de I'hépatite $\mathrm{C}(\mathrm{VHC})$ peut être difficile à détecter, car l'infection peut demeurer asymptomatique pendant des décennies. Souvent, les personnes atteintes ne se voient pas offrir ni ne recherchent de test de dépistage jusqu'à ce que les symptômes se manifestent. Cet état de fait souligne l'importance d'accroître la conscience et les connaissances auprès des fournisseurs de soins de santé et de la population en général afin d'atteindre les objectifs concernant l'hépatite virale.

Objectifs : Réaliser une recherche exploratoire de la documentation afin de caractériser l'état actuel de la conscience et des connaissances des fournisseurs de soins de santé et de la population en général en ce qui a trait aux infections au VHC, à sa transmission, à sa prévention et à son traitement ainsi que de reconnaître les lacunes dans les connaissances qu'un plan d'action de santé publique pourrait corriger.

Méthodologie : Une recherche documentaire a été effectuée à l'aide d'Embase, de Medline et de Scopus afin de trouver des études publiées entre janvier 2012 et juillet 2017 . Une recherche de la documentation parallèle a aussi été entreprise. Les données suivantes ont été extraites : le nom de l'auteur, l'année de publication, le modèle des études, la population, le milieu, le pays, la méthode de collecte des données et les résultats en matière de conscience et de connaissance. Les commentaires, le courrier des lecteurs et les revues narratives ont été exclus.

Résultats : Dix-neuf études ont été intégrées à la présente recherche exploratoire de la documentation. La définition de "conscience » et de "connaissance " variait entre les études; à certains moments, ces termes étaient utilisés de manière interchangeable Les fournisseurs de soins de santé ont plus fréquemment ciblé la consommation de drogues injectables ou les transfusions sanguines comme des voies de transmission du VHC que les autres voies de transmission comme le tatouage utilisant des outils non stériles et la transmission sexuelle. Parmi la population en général, les idées fausses concernant le VHC comprenaient la croyance que le fait de s'embrasser ou d'avoir de simples contacts était des voies de transmission du virus et qu'un vaccin pouvant prévenir l'infection était disponible. Globalement, des lacunes existaient dans les données concernant d'autres populations à haut risque (p. ex., les Autochtones, les personnes incarcérées).

Conclusion : Des campagnes d'information publique et des campagnes d'éducation professionnelle sur le VHC pourraient aider à soutenir le dépistage du VHC fondé sur le risque. De futurs travaux de recherche pourraient évaluer la sensibilisation d'autres populations présentant un risque accru et introduire des définitions uniformes de la «conscience» et de la «connaissance».

Citation proposée : Ha S, Timmerman K. Conscience et connaissance de l'hépatite C chez les fournisseurs de soins de santé et la population en général : une recherche exploratoire de la documentation. Relevé des maladies transmissibles au Canada 2018;44(7/8):175-85. https://doi.org/10.14745/ccdr.v44i78a02f

Mots-clés : conscience, connaissance, hépatite $C$, revue exploratoire de la documentation, fournisseurs de soins de santé

\section{Affiliation}

${ }^{1}$ Centre de la lutte contre les maladies transmissibles et les infections, Agence de la santé publique du Canada, Ottawa (Ontario)

Correspondance : shalane.ha@ canada.ca 


\section{Contexte}

Le virus de l'hépatite $\mathrm{C}(\mathrm{VHC})$ cause une inflammation du foie qui peut devenir chronique. L'infection chronique au VHC peut être asymptomatique pendant des décennies avant que les symptômes se manifestent. Globalement, environ 71 millions de personnes sont atteintes d'une infection chronique au VHC (1). L'infection chronique ne se détecte pas facilement; même lorsque les symptômes se manifestent, ils sont souvent non spécifiques (p. ex., de la fatigue) (2). L'infection chronique au VHC peut mener à une cirrhose ou au cancer du foie. Environ un demi-million de personnes meurent chaque année de maladies du foie liées au VHC (3).

En 2011, environ 220000 à 246000 personnes étaient atteintes d'une infection chronique au VHC au Canada et approximativement $44 \%$ d'entre elles ignoraient qu'elles étaient porteuses de l'infection (4). Au cours des dernières années, de grands progrès ont été réalisés dans le traitement du VHC et l'infection est maintenant guérissable. Les programmes de traitement antérieurs étaient fondés sur le PEG-Interféron et la ribavirine qui nécessitaient de plus longues durées de traitement et comportaient davantage d'effets indésirables. Les nouveaux traitements antiviraux à action directe (AAD) sans interférons se sont montrés hautement efficaces et présentent moins d'effets indésirables. Actuellement, la plupart des formulaires provinciaux et territoriaux couvrent ces nouveaux traitements et le Canada a commencé à observer une diminution des hospitalisations associées à l'infection au VHC et à la maladie hépatique chronique (5).

En 2016, la 69 Assemblée mondiale de la santé a adopté la Stratégie mondiale du secteur de la santé sur l'hépatite virale dans le but d'éliminer d'ici 2030 l'hépatite B et l'hépatite $C$ en tant que menaces à la santé publique. (6). Le but est d'obtenir un diagnostic pour $90 \%$ des cas d'hépatite virale $B$ et $C$ et de traiter $80 \%$ des personnes atteintes des infections chroniques au virus de I'hépatite $B(\mathrm{VHB})$ et au VHC (6). La conscience et la connaissance de l'hépatite $C$ constituent un premier pas important de la stratégie d'élimination. L'identification du VHC au moyen d'un test de dépistage est essentielle aux patients afin qu'ils modifient de manière appropriée leurs habitudes de vie et qu'ils commencent leur traitement.

Une conscience et des connaissances limitées en ce qui a trait au VHC ont été ciblées comme les obstacles principaux des fournisseurs de soins de santé qui offrent le test de dépistage de I'hépatite $\mathrm{C}$ et pour les patients qui cherchent à l'obtenir (7). $\mathrm{Ce}$ manque a pour conséquences la poursuite de la transmission du $\mathrm{VHC}$ et des occasions manquées de prévention et de traitement. Dans un effort pour améliorer le dépistage fondé sur les risques au Canada et réduire le nombre de personnes qui ignorent être infectées, il est important de comprendre l'état actuel de la conscience et de la connaissance du VHC à la fois chez les fournisseurs de soins de santé que dans la population en général.

Les objectifs de la présente recherche documentaire sont de résumer l'état de la conscience et des connaissances des fournisseurs de soins de santé et de la population en général sur I'histoire naturelle du VHC et sur sa transmission, sa prévention et son traitement, et de relever au sein de ces deux groupes les lacunes dans les connaissances qui pourraient être corrigées par des plans d'action de santé publique.

\section{Méthodologie}

Nous avons travaillé avec un bibliothécaire de recherche afin de réaliser une recherche documentaire dans Embase, Medline et Scopus sur la conscience et la connaissance du VHC chez les fournisseurs de soins de santé et la population en général. Nous avons aussi cherché dans la documentation parallèle (c.-à-d. dans les rapports disponibles dans le domaine public) en utilisant le moteur de recherche Google. Les termes de recherche suivants ont été utilisés : hépatite $\mathrm{C}, \mathrm{VHC}$, conscience et connaissance. Les études étaient retenues pour la recherche documentaire si elles étaient publiées entre janvier 2012 et juillet 2017, en français ou en anglais, qu'elles étaient réalisées au Canada ou dans des pays économiquement développés et dotés de ressources similaires, et qu'elles portaient sur la population en général ou les fournisseurs de soins de santé. Nous avons restreint la recherche aux cinq dernières années afin de saisir les plus récentes données. Les commentaires, le courrier des lecteurs et les études de cas ont été exclus. Les résultats d'intérêt comprennent la conscience et la connaissance du VHC qui sont définies de différente façon selon l'étude.

Après avoir trié les titres et des résumés des articles potentiellement pertinents, nous avons examiné l'ensemble du texte des études retenues. Nous avons élaboré des formulaires d'extraction de données et extrait les données suivantes : le nom de l'auteur, l'année de publication, le modèle des études, la population, le milieu, le pays, la méthode de collecte des données et les résultats en matière de connaissance et de conscience.

Afin de procéder à une recherche exploratoire de la documentation, on a réalisé une analyse qualitative des données recueillies et regroupé les résultats par thème, mais on n'a pas mené d'évaluation détaillée de la qualité globale ou du risque de biais.

\section{Résultats}

La recherche documentaire a relevé 141 articles potentiellement pertinents sur la conscience et la connaissance du VHC par les fournisseurs de soins de santé et la population en général. Une recherche manuelle des listes de références a trouvé cinq références additionnelles. Trois autres rapports ont été ciblés par une recherche de la documentation parallèle. Après avoir trié le titre et le résumé et procédé à l'examen détaillé des textes ciblés, on a retenu 19 études pour la présente recherche exploratoire de la documentation (figure 1).

À certains moments, les termes "conscience» et "connaissance» étaient utilisés de manière interchangeable dans les études retenues. La conscience était définie comme soit le fait de savoir qu'on est infecté au VHC ou qu'on est séropositif, ou de connaître son diagnostic, soit la conscience de l'existence du VHC, de ses facteurs de risque ou de la disponibilité d'un traitement. La connaissance, pour sa part, pouvait signifier I'histoire naturelle et les conséquences du VHC, les facteurs de 
risque du virus et ses voies de transmission ou la disponibilité d'un vaccin et d'un traitement. Par conséquent, on cite les résultats selon la façon dont les études elles-mêmes définissent ces termes.

Figure 1 : Organigramme du processus de sélection des études

\begin{tabular}{|c|c|}
\hline $\begin{array}{l}\text { Notices repérées par: } \\
\text { EMBASE }(n=52) \\
\text { Medline }(n=40)\end{array}$ & \\
\hline $\begin{array}{l}\text { Scopus }(n=15) \\
\text { Fouille manuelle }(n=3)\end{array}$ & \multirow{4}{*}{$\begin{array}{l}\text { Raisons d'exclusion }(n=94) \text { : } \\
\text { Objectif non centré sur les } \\
\text { obstacles et les facteurs qui } \\
\text { facilitent }(n=50) \\
\text { Ne répondait pas aux critères du } \\
\text { modèle de l'étude ( } n=29) \\
\text { Ne ciblait pas les patients ou les } \\
\text { fournisseurs de soins de santé }\end{array}$} \\
\hline Nombre total de notices $(n=110)$ & \\
\hline & \\
\hline $\begin{array}{l}\text { Nombre total de notices répondant } \\
\text { aux critères d'inclusion }(n=16)\end{array}$ & \\
\hline
\end{tabular}

Abréviations : n, nombre; PCDI, personne qui consomme des drogues injectables; VHC, virus de I'hépatite C

\section{Caractéristiques des études retenues}

La majorité des études retenues ont été réalisées aux États-Unis (É.-U.; $n=8)$, suivis par le Canada $(n=5)$ et l'Australie $(n=3)$. Les autres études provenaient de l'Allemagne, de l'Italie, du Japon et des Pays-Bas. La plupart des études $(n=13)$ ciblaient la population en général et moins du tiers $(n=5)$ portaient sur les fournisseurs de soins de santé; une étude comprenait les deux populations. Les participants étaient recrutés de différents milieux dont des centres hospitaliers, des cliniques de consultation externe, des établissements de soins primaires, des services d'urgence et des panels en ligne. Les méthodes de collecte de données comprenaient souvent des questionnaires remplis en ligne, en personne ou au téléphone. (Pour de plus amples détails sur les études retenues, consultez l'appendice 1.)

Dans les études qui portaient sur les fournisseurs de soins de santé, les catégories d'emploi comprenaient des médecins, des infirmiers, des résidents, des étudiants en art dentaire et des spécialistes (p. ex., des hépatologues et des gastroentérologues). Parmi les études qui ne portaient pas sur des fournisseurs de soins de santé, les groupes de population comprenaient des personnes infectées au $\mathrm{VHC}$, avec ou sans la présence d'une infection au $\mathrm{VIH}$, des hommes qui ont des relations sexuelles avec d'autres hommes, des immigrants, la population en général et les adultes nés entre 1945 et 1965 (tableau 1).

\section{Conscience}

Six études portaient sur la conscience de I'hépatite C $(11,12,14,17,18,24)$. Les types de conscience variaient entre ces études : conscience des facteurs de risque, conscience du traitement, conscience de son infection et conscience de l'existence du VHC. Quatre études comprenaient des résultats portant sur la conscience du VHC par la population en général $(11,12,14,17)$, une sur la conscience du VHC par les hommes qui ont des relations sexuelles avec d'autres hommes (18) et une sur la conscience du traitement par des fournisseurs canadiens de soins de santé (24).
Tableau 1 : Résumé des études retenues

\begin{tabular}{|c|c|}
\hline Caractéristiques & Nombre d'études $(n)^{a}$ \\
\hline \multicolumn{2}{|l|}{ Pays } \\
\hline É.-U. & 8 \\
\hline Canada & 5 \\
\hline Australie & 3 \\
\hline Pays-Bas & 2 \\
\hline Allemagne & 1 \\
\hline Italie & 1 \\
\hline Japon & 1 \\
\hline Autre & 3 \\
\hline \multicolumn{2}{|l|}{ Fournisseurs de soins de santé } \\
\hline Médecins & 3 \\
\hline Infirmiers & 3 \\
\hline $\begin{array}{l}\text { Médecins spécialistes (hépatologues, } \\
\text { gastroentérologues, etc.) }\end{array}$ & 2 \\
\hline Étudiants en médecine & 1 \\
\hline Autre & 1 \\
\hline \multicolumn{2}{|c|}{ Personnes autres que les fournisseurs de soins de santé } \\
\hline $\begin{array}{l}\text { Personnes atteintes du VHC, avec ou } \\
\text { sans co-infection au VIH }\end{array}$ & 4 \\
\hline $\begin{array}{l}\text { Hommes qui ont des relations sexuelles } \\
\text { avec d'autres hommes }\end{array}$ & 3 \\
\hline Population en général & 2 \\
\hline Personnes nées entre 1945-1965 & 2 \\
\hline Immigrants & 1 \\
\hline Autre & 1 \\
\hline
\end{tabular}

Abréviations : $n$, nombre ; $\mathrm{VHC}$, virus de l'hépatite $\mathrm{C} ; \mathrm{VIH}$, virus de l'immunodéficience humaine ${ }^{a}$ Certaines études comprenaient plus d'une population ou plus d'un pays

Deux études ont déterminé que la population en général était un peu sensibilisée à l'existence de l'hépatite $C(11,17)$. Comparativement à la population en général (27\%), les enfants de la génération du baby-boom nés au Canada étaient plus susceptibles de savoir que les consommateurs de drogues injectables présentent un risque accru d'être atteints du VHC (14). Cependant, les résultats du National Health and Nutrition Examination Survey (NHANES) des États-Unis mentionnaient que moins de la moitié des Américains atteints d'une infection au VHC en étaient conscients (12). Deux études ont déterminé que la population en général ne comprenait pas clairement la différence entre les hépatites $A, B$ et $C(11,19)$.

\section{Connaissance}

Toutes les études retenues ont évalué la connaissance du VHC. La connaissance a été mesurée à l'aide d'une série de questionnaires où le répondant devait répondre à des énoncés oui/non/je-ne-sais-pas ou à des énoncés vrais ou faux. II pouvait aussi évaluer lui-même son niveau de connaissance. La connaissance a été évaluée sur les thèmes suivants : I'histoire naturelle du VHC, les voies de transmission et la disponibilité d'un vaccin ou de traitements. 


\section{L'histoire naturelle du VHC et ses conséquences}

Trois études comprenaient des renseignements sur la connaissance des fournisseurs de soins de santé concernant I'histoire naturelle et les conséquences du VHC $(16,25,26)$. Dans une étude sur les médecins canadiens, $35 \%$ ont mentionné "en connaître beaucoup» sur les symptômes associés au VHC (16). Dans une petite étude d'étudiants en art dentaire de la Bulgarie, $80 \%$ ont déclaré savoir que l'infection au VHB ou au VHC pouvait être asymptomatique (26). De plus, les résidents, les médecins, les infirmiers praticiens et les adjoints au médecin travaillant aux services des urgences aux États-Unis ont mentionné bien connaître les manifestations du VHC (pourcentage non cité) (25).

Huit études comportaient des renseignements sur les connaissances de la population en général concernant l'histoire naturelle du VHC $(8,9,11,13,14,16,17,19)$. Deux études canadiennes ont démontré que de 83 à $90 \%$ des participants savaient que les personnes atteintes du VHC pouvaient ignorer être porteuses d'une infection $(14,16)$. De même, plus de la moitié (57\%) des personnes de la génération du baby-boom savaient que le $\mathrm{VHC}$ pouvait causer le cancer du foie et $61 \%$ croyaient qu'une personne atteinte d'une infection au VHC peut ne pas présenter de symptômes (8). Une étude mentionnait qu'un tiers des hommes qui ont des relations sexuelles avec $\mathrm{d}^{\prime}$ autres hommes savaient que l'infection au VHC pouvait mener au cancer du foie (31\%) et à une insuffisance hépatique (37\%) (18). À l'inverse, une étude internationale réalisée auprès d'immigrants asiatiques a fait remarquer qu'il existait une confusion entre les différents types d'infection hépatique et une incertitude à propos de l'histoire naturelle de l'infection (19).

\section{Connaissance de la transmission}

Deux études ont porté sur la connaissance des fournisseurs de soins de santé sur la transmission du VHC $(22,26)$. La majorité des fournisseurs de soins de santé interrogés dans les études ont défini les principales voies de transmission comme étant les transfusions de sang, l'exposition à du sang lors de l'activité sexuelle et l'échange d'aiguilles lors de la consommation de drogues injectables $(22,26)$. Un petit pourcentage $(12 \%)$ des infirmiers travaillant aux cliniques d'hémodialyse en Italie croyaient, à tort, que le VHC pouvait être transmis par un baiser et $19 \%$ ne savaient pas que se faire tatouer pouvait être une voie de transmission du virus (22).

Dix études ont présenté des renseignements sur la connaissance de la transmission du VHC chez la population en général (8, 10-12,14-17,19,21). Selon une étude canadienne, les voies de transmission les plus souvent connues seraient les transfusions de sang, les relations sexuelles non sécuritaires et non protégées, et la consommation de drogue injectable à l'aide d'aiguilles souillées ou qui ont été partagées (14). Peu de Canadiens ont nommé d'autres voies de transmission comme l'échange $d$ 'articles d'hygiène personnelle (7\%), se faire tatouer ou obtenir un perçage corporel (4\%), l'exposition à des facteurs de risques lors de voyage dans des pays étrangers où le VHC est endémique ( $4 \%$ ) et la transmission de la mère à l'enfant pendant la grossesse (1\%) (14). Par ailleurs, environ 54 à $62 \%$ de la population canadienne savait que le VHC se transmet principalement par contact direct de sang à sang (16). Dans quatre études, un petit pourcentage de la population en général a indiqué que le $\mathrm{VHC}$ peut être transmis par un baiser ou par simple contact $(8,12,14,21)$.

\section{Connaissance du traitement}

Deux études récentes, publiées après l'introduction des nouveaux traitements antiviraux à action directe (AAD) sans interférons, se sont concentrées sur la capacité de guérir du $\mathrm{VHC}(8,24)$

Parmi les fournisseurs de soins de santé, les spécialistes (c.-à-d. les hépatologues, les gastroentérologues et les infirmiers en hépatologie) ont obtenu de meilleurs résultats sur les énoncés de connaissance portant sur les traitements du VHC que les médecins généralistes $(23,24)$. Parmi les dix médecins de première ligne interrogés, sept étaient incertains ou ne connaissaient pas les nouveaux AAD sans interférons et connaissaient mal leurs mécanismes d'action (24).

Aux États-Unis, 51 \% des personnes de la génération du baby-boom se présentant au service des urgences croyaient, avec raison, que le $\mathrm{VHC}$ était guérissable et $77 \%$ savaient que de nouveaux médicaments étaient disponibles pour le traiter (8). Par contre, trois études ont détecté parmi la population en général une idée fausse quant à la disponibilité d'un vaccin pour prévenir les infections au VHC $(11,15,21)$. Environ la moitié des Canadiens interrogés (50 \%) dans une étude croyaient qu'il existait un vaccin pour prévenir I'infection au VHC (14). Dans deux études américaines, $42 \%$ des Américains de la génération du baby-boom et $60 \%$ des Afro-Américains appartenant à la même génération partageaient leur opinion $(8,11)$.

Un résumé des conclusions est présenté au tableau 2.

\section{Tableau 2 : Résumé des conclusions sur la conscience et la connaissance du virus de l'hépatite $C$ parmi les professionnels de la santé et la population en général}

\begin{tabular}{|l|l|}
\hline \multicolumn{1}{|c|}{ Résultats } & \multicolumn{1}{c|}{ Conclusions clés } \\
\hline Conscience & $\begin{array}{l}\text { Population en général : } \\
\text { La population en général était consciente du VHC et } \\
\text { de ses principaux facteurs de risque (14) } \\
\text { Les hommes qui ont des relations sexuelles avec } \\
\text { d'autres hommes étaient très conscients de l'existence } \\
\text { du traitement contre le VHC (18) }\end{array}$ \\
\hline Connaissance & $\begin{array}{l}\text { Fournisseurs de soins de santé : } \\
\text { Les médecins spécialistes étaient plus au courant des } \\
\text { nouveaux traitements contre le VHC que les médecins } \\
\text { de première ligne (24) } \\
\text { Les fournisseurs de soins de santé connaissaient moins } \\
\text { certaines voies de transmission du VHC (p. ex., les } \\
\text { pratiques non sécuritaires de tatouage et de perçage } \\
\text { corporel) comparativement aux voies principales (c.- } \\
\text { à-d. la consommation de drogues injectables) (22,26) } \\
\text { Population en général : } \\
\text { La population en général entretenait des idées fausses } \\
\text { à propos des facteurs de risque de transmission de } \\
\text { I'hépatite C (p. ex. par simple contact, par la salive, par } \\
\text { un baiser) (11,12,14,16,19) } \\
\text { II y avait aussi des idées fausses sur la disponibilité } \\
\text { d'un vaccin (8,14,16) } \\
\text { Globalement, le traitement de I'hépatite C par les } \\
\text { AAD sans interférons était peu connu (8,9,13,14,16) }\end{array}$ \\
\hline
\end{tabular}

Abréviations : $A A D$, antiviraux à action directe; $\mathrm{VHC}$, virus de l'hépatite $\mathrm{C}$ 


\section{Discussion}

Autant que nous sachions, il s'agit de la première recherche exploratoire de la documentation qui présente le portrait de l'état de la connaissance des fournisseurs de soins de santé et de la population en général sur le VHC. Globalement, les fournisseurs de soins de santé connaissent les voies de transmission et les facteurs de risque les plus fréquents, alors que les spécialistes sont mieux au fait des traitements que les médecins de première ligne $(23,24)$. La population en général est sensibilisée au VHC. Cependant, certaines personnes ne connaissent pas la différence entre les hépatites $A, B$ et $C$, ont des idées fausses sur les voies de transmission et croient incorrectement qu'un vaccin préventif contre le VHC existe.

Certaines limites doivent être prises en considération lorsqu'on interprète nos conclusions. Tout d'abord, il existe une lacune dans l'uniformisation des définitions de "connaissance» et de "conscience» et les termes étaient souvent utilisés de manière interchangeable. Deuxièmement, seules quelques études se sont penchées sur la conscience et la connaissance des AAD sans interférons. Finalement, les conclusions étaient fondées sur des études transversales qui ne saisissent les données que de la population étudiée à un point donné dans le temps.

De futurs travaux de recherche pourraient évaluer des populations à haut risque (p. ex., les populations autochtones ou les personnes incarcérées), intégrer des définitions claires et uniformes de "conscience » et de "connaissance » et évaluer les facteurs qui peuvent être associés à des différences de conscience et de connaissances (p. ex., le milieu rural par rapport au milieu urbain et le statut socioéconomique). Des travaux additionnels sur la connaissance du VHC chez les fournisseurs de services de santé pourraient aussi aider à adapter de futurs transferts de connaissance et d'échanges de produits.

En conclusion, augmenter la conscience et la connaissance des fournisseurs de soins de santé et de la population en général sur le VHC peut faciliter la discussion sur le besoin d'avoir recours à son dépistage. Les conclusions et les lacunes ciblées dans la présente recherche exploratoire peuvent aider à éclairer de futures interventions et de prochaines campagnes de santé publique en lien avec le VHC et soutenir la Stratégie mondiale du secteur de la santé sur l'hépatite virale.

\section{Déclaration des auteurs}

S. H. - Conceptualisation, méthodologie, rédaction (version finale), conservation des données, validation, analyse formelle, rédaction, examen et révision, supervision, administration du projet, visualisation

K. T. - Conceptualisation, méthodologie, examen et révision, supervision, administration du projet, visualisation

\section{Conflit d'intérêts}

Aucun.

\section{Remerciements}

Nous voudrions remercier la $D^{r e}$ Margaret Gale-Rowe et le $D^{r}$ Jun Wu pour leurs contributions à la conceptualisation et à la révision du présent manuscrit, Audréanne Garand pour son soutien à la collecte des données, à leur extraction et à l'analyse initiale des résultats ainsi qu'au bibliothécaire de Santé Canada qui a aidé à réaliser la recherche documentaire.

\section{Financement}

Le présent travail a été soutenu par l'Agence de la santé publique du Canada.

\section{Références}

1. Organisation mondiale de la Santé. Hépatite C: principaux faits. Geneva: Organisation mondiale de la Santé; 2017. http://www.who. int/fr/news-room/fact-sheets/detail/hepatitis-c

2. Westbrook RH, Dusheiko G. Natural history of hepatitis C. J Hepatol 2014 Nov;61(1 Suppl):S58-68. http://dx.doi.org/10.1016/j. jhep.2014.07.012 PubMed (https://www.ncbi.nlm.nih.gov/ pubmed/25443346)

3. Organisation mondiale de la Santé. Global hepatitis report, 2017. Geneva: Organisation mondiale de la Santé; 2017. http://apps. who.int/iris/bitstream/handle/10665/255016/9789241565455-eng. pdf? sequence=1

4. Trubnikov M, Yan P, Archibald C. Estimation de la prévalence de l'infection par le virus de l'hépatite $C$ au Canada, 2011. Relevé des maladies transmissibles au Canada 2014 Déc;40(19):429-36. PubMed (https://www.ncbi.nlm.nih.gov/pubmed/29769874)

5. Schanzer D, Pogany L, Aho J, Tomas K, Gale-Rowe M, Kwong JC, Janjua NZ, Feld J. Répercussions des antiviraux à action directe contre l'hépatite $\mathrm{C}$ sur les taux d'hospitalisation au Canada, de 2012 à 2016. Relevé des maladies transmissibles au Canada 2018;44(7/8):167-74. https://www.canada.ca/fr/sante-publique/ services/rapports-publications/releve-maladies-transmissiblescanada-rmtc/numero-mensuel/2018-44/numero-7-8-5-juillet-2018/ article-1-taux-hospitalisation-canadiens-hepatite-c.html

6. Organisation mondiale de la Santé. Stratégie mondiale du secteur de la santé contre l'hépatite virale, 2016-2021. Geneva: Organisation mondiale de la Santé; 2016. http://www.who.int/hepatitis/ strategy2016-2021/ghss-hep/fr/

7. McLeod A, Cullen BL, Hutchinson SJ, Roy KM, Dillon JF, Stewart EA, Goldberg DJ. Limited impact of awareness-raising campaigns on hepatitis $\mathrm{C}$ testing practices among general practitioners. J Viral Hepat 2017 Nov;24(11):944-54. http://dx.doi.org/10.1111/jvh.12724 PubMed (https://www.ncbi.nlm.nih.gov/pubmed/28502088)

8. Allison WE, Chiang W, Rubin A, Oshva L, Carmody E. Knowledge about hepatitis $C$ virus infection and acceptability of testing in the 1945-1965 birth cohort (baby boomers) presenting to a large urban emergency department: a pilot study. J Emerg Med 2016 Jun;50(6):825-831.e2. http://dx.doi.org/10.1016/j. jemermed.2016.02.001 PubMed (https://www.ncbi.nlm.nih.gov/ pubmed/26954104)

9. CATIE. Room for improvement: knowledge exchange needs of people living with hepatitis C. Toronto: CATIE; 2015. http:// www.catie.ca/sites/default/files/Hepatitis\%20C\%20needs\%20 assessment\%20report_final.pdf

10. Chen EY, North CS, Fatunde O, Bernstein I, Salari S, Day B, Jain MK. Knowledge and attitudes about hepatitis $C$ virus (HCV) infection and its treatment in $\mathrm{HCV}$ mono-infected and HCV/HIV co-infected adults. J Viral Hepat 2013 Oct;20(10):708-14. http://dx.doi. 
org/10.1111/jvh.12095 PubMed (https://www.ncbi.nlm.nih.gov/ pubmed/24010645)

11. Crutzen R, Göritz AS. Public awareness and practical knowledge regarding Hepatitis A, B, and C: a two-country survey. J Infect Public Health 2012 Apr;5(2):195-8. http://dx.doi.org/10.1016/j. jiph.2011.12.001 PubMed (https://www.ncbi.nlm.nih.gov/ pubmed/22541268/)

12. Denniston MM, Klevens RM, McQuillan GM, Jiles RB. Awareness of infection, knowledge of hepatitis $C$, and medical follow-up among individuals testing positive for hepatitis C: National Health and Nutrition Examination Survey 2001-2008. Hepatology 2012 Jun;55(6):1652-61. http://dx.doi.org/10.1002/hep.25556 PubMed (https://www.ncbi.nlm.nih.gov/pubmed/22213025)

13. Eguchi $\mathrm{H}$, Wada K. Knowledge of HBV and HCV and individuals' attitudes toward HBV- and HCV-infected colleagues: a national cross-sectional study among a working population in Japan. PLoS One 2013 Sep;8(9):e76921. http://dx.doi.org/10.1371/ journal.pone.0076921 PubMed (https://www.ncbi.nlm.nih.gov/ pubmed/24086765)

14. EKOS Research Associates Inc. Sondage de suivi de 2012 sur les attitudes touchant le VIH/sida. Ottawa: EKOS; 2012 Oct. http://www. catie.ca/sites/default/files/2012-HIV-AIDS-attitudinal-tracking-surveyfinal-report.pdf

15. Hopwood M, Lea T, Aggleton P. Multiple strategies are required to address the information and support needs of gay and bisexual men with hepatitis C in Australia. J Public Health (Oxf) 2016 Mar;38(1):156-62. http://dx.doi.org/10.1093/pubmed/fdv002 PubMed (https://www.ncbi.nlm.nih.gov/pubmed/25626415)

16. Ipsos Healthcare. Survey on hepatitis $C$ knowledge and perception among Canadians and GP, September 2012. Paris: Ipsos; 2012. https://www.ipsos.com/sites/default/files/publication/2013-01/5977report.pdf

17. Lambers FA, Prins M, Davidovich U, Stolte IG. High awareness of hepatitis $\mathrm{C}$ virus (HCV) but limited knowledge of $\mathrm{HCV}$ complications among HIV-positive and HIV-negative men who have sex with men. AIDS Care 2014 Apr;26(4):416-24. http://dx.doi.org/10.1080/0 9540121.2013.832721 PubMed (https://www.ncbi.nlm.nih.gov/ pubmed/24024525)

18. Lea T, Hopwood M, Aggleton P. Hepatitis $C$ knowledge among gay and other homosexually active men in Australia. Drug Alcohol Rev 2016 Jul;35(4):477-83. http://dx.doi.org/10.1111/dar.12333 PubMed (https://www.ncbi.nlm.nih.gov/pubmed/26369759)
19. Owiti JA, Greenhalgh T, Sweeney L, Foster GR, Bhui KS. Illness perceptions and explanatory models of viral hepatitis $B$ \& $C$ among immigrants and refugees: a narrative systematic review. BMC Public Health 2015 Feb;15:151. http://dx.doi.org/10.1186/s12889-0151476-0 PubMed (https://www.ncbi.nlm.nih.gov/pubmed/25886390)

20. Pundhir P, North CS, Fatunde O, Jain MK. Health beliefs and co-morbidities associated with appointment-keeping behavior among HCV and HIV/HCV patients. J Community Health 2016 Feb;41(1):30-7. http://dx.doi.org/10.1007/s10900-015-0059-4 PubMed (https://www.ncbi.nlm.nih.gov/pubmed/26179172)

21. Rashrash ME, Maneno MK, Wutoh AK, Ettienne EB, Daftary MN. An evaluation of hepatitis $C$ knowledge and correlations with health belief model constructs among African American "baby boomers". J Infect Public Health 2016 Jul-Aug;9(4):436-42. http://dx. doi. org/10.1016/j.jiph.2015.11.005 PubMed (https://www.ncbi.nlm.nih. gov/pubmed/26706773)

22. Bianco A, Bova F, Nobile CG, Pileggi C, Pavia M; Collaborative Working Group. Healthcare workers and prevention of hepatitis $C$ virus transmission: exploring knowledge, attitudes and evidence-based practices in hemodialysis units in Italy. BMC Infect Dis 2013 Feb;13(76):76. http://dx.doi.org/10.1186/1471-2334-13-76 PubMed (https://www.ncbi.nlm.nih.gov/pubmed/23391009)

23. McGowan CE, Monis A, Bacon BR, Mallolas J, Goncales FL, Goulis I, Poordad F, Afdhal N, Zeuzem S, Piratvisuth T, Marcellin P, Fried MW. A global view of hepatitis C: physician knowledge, opinions, and perceived barriers to care. Hepatology 2013 Apr;57(4):1325-32. http://dx.doi.org/10.1002/hep.26246 PubMed (https://www.ncbi. nlm.nih.gov/pubmed/23315914)

24. Naghdi R, Seto K, Klassen C, Emokpare D, Conway B, Kelley M, Yoshida E, Shah HA. A hepatitis C educational needs assessment of Canadian healthcare providers. Can J Gastroenterol Hepatol 2017 10:1-10. https://doi.org/10.1155/2017/5324290

25. Rotte M, O'Donnell R. Knowledge, beliefs, and attitudes of emergency department health care providers towards hepatitis $C$ and rapid hepatitis C testing. Ann Emerg Med 2013;62(4):S103. http://dx.doi.org/10.1016/j.annemergmed.2013.07.108

26. Todorova TT, Tsankova G, Tsankova D, Kostadinova T, Lodozova $\mathrm{N}$. Knowledge and attitude towards hepatitis $B$ and hepatitis $C$ among dental medicine students. J of IMAB 2015;21(3):810-3. http://dx.doi.org/10.5272/jimab.2015213.810 
Appendice 1 : Description des études retenues $(n=19)$

\begin{tabular}{|c|c|c|c|}
\hline $\begin{array}{c}\text { Auteurs(s), année } \\
\text { de publication et } \\
\text { pays }\end{array}$ & $\begin{array}{l}\text { Modèle de l'étude, } \\
\text { population et lieu }\end{array}$ & $\begin{array}{l}\text { Méthode de } \\
\text { la collecte de } \\
\text { données }\end{array}$ & Résultats et conclusions \\
\hline \multicolumn{4}{|c|}{ Population en général $(n=14)$} \\
\hline $\begin{array}{l}\text { Allison et coll. (2016) (8) } \\
\text { É.-U. }\end{array}$ & $\begin{array}{l}\text { Étude transversale } \\
\text { Génération du baby-boom } \\
(1945-1965)(n=915) \\
\text { Service des urgences en } \\
\text { milieu urbain }\end{array}$ & $\begin{array}{l}\text { Entrevues structurées } \\
\text { dans les six semaines } \\
\text { suivant un test } \\
\text { de détection des } \\
\text { anticorps du VHC } \\
\text { afin d'évaluer la } \\
\text { connaissance }\end{array}$ & $\begin{array}{l}\text { Connaissances } \\
\text { - La plupart des participants connaissaient l'histoire naturelle et les } \\
\text { complications de l'infection au VHC } \\
\text { - La plupart des participants connaissaient les facteurs de risque du } \\
\text { VHC } \\
\text { - Certains participants avaient des idées fausses sur la transmission } \\
\text { (c.-à-d. échanger un baiser) } \\
\text { - Plus de la moitié pensaient qu'un vaccin préventif contre le VHC } \\
\text { - existait } \\
\text { - Plusieurs ne savaient pas que le VHC est guérissable }\end{array}$ \\
\hline $\begin{array}{l}\text { CATIE (2015) (9) } \\
\text { Canada }\end{array}$ & $\begin{array}{l}\text { Étude transversale } \\
\text { Personnes atteintes du } \\
\text { VHC }(n=326) \\
\text { Cliniques médicales } \\
\text { offrant des soins aux } \\
\text { personnes infectées par } \\
\text { le VHC }\end{array}$ & $\begin{array}{l}\text { Questionnaire } \\
\text { autoadministré } \\
\text { (versions papier et en } \\
\text { ligne) }\end{array}$ & $\begin{array}{l}\text { Connaissance } \\
\text { - } 23 \% \text { ont déclaré connaître beaucoup de choses sur l'hépatite C } \\
\text { - Les jeunes répondants, les hommes et les Autochtones étaient } \\
\text { plus susceptibles de déclarer de plus faibles niveaux de } \\
\text { connaissance sur l'hépatite C } \\
\text { - } 20 \% \text { ont déclaré connaître beaucoup de choses sur le traitement } \\
\text { - Les jeunes répondants et les Autochtones étaient plus } \\
\text { susceptibles de déclarer de plus faibles niveaux de connaissance } \\
\text { sur l'hépatite C }\end{array}$ \\
\hline $\begin{array}{l}\text { Chen et coll. (2013) (10) } \\
\text { É.-U. }\end{array}$ & $\begin{array}{l}\text { Étude transversale } \\
\text { Infection au VHC et } \\
\text { co-infection au VIH/VHC } \\
(\mathrm{n}=292) \\
\text { Consultations externes }\end{array}$ & $\begin{array}{l}\text { Enquête transversale } \\
\text { et enquêtes } \\
\text { précédant et suivant } \\
\text { la formation }\end{array}$ & $\begin{array}{l}\text { Connaissances } \\
\text { - Globalement, la connaissance du VHC était limitée, moins de } \\
50 \text { \% des questions ayant reçu une bonne réponse } \\
\text { - Aucune différence entre les groupes vivant avec une seule } \\
\text { infection et ceux vivant avec plusieurs infections concernant le } \\
\text { degré de connaissance du VHC ou les sous-échelles représentant } \\
\text { la maladie et la transmission du VHC } \\
\text { - Les participants co-infectés présentaient une plus grande } \\
\text { connaissance moyenne du traitement contre le VHC } \\
\text { comparativement aux participants du groupe vivant avec une } \\
\text { seule infection }\end{array}$ \\
\hline $\begin{array}{l}\text { Crutzen et Goritz } \\
(2012)(11) \\
\text { Allemagne et Pays-Bas }\end{array}$ & $\begin{array}{l}\text { Étude transversale } \\
\text { Population en général } \\
\text { en Allemagne }(n=1989) \\
\text { et aux Pays-Bas }(n=668) \\
\text { Panel en ligne }\end{array}$ & $\begin{array}{l}\text { Deux enquêtes } \\
\text { à grande échelle } \\
\text { administrées à des } \\
\text { panels en ligne }\end{array}$ & $\begin{array}{l}\text { Conscience } \\
\text { - Forte sensibilisation à l'hépatite A, B et C (aucune valeur de } \\
\text { pourcentage ou d'échelles fournies) } \\
\text { Connaissances } \\
\text { - La connaissance était très faible dans les deux pays (légèrement } \\
\text { au-dessus de } 50 \% \text { de bonnes réponses) } \\
\text { - Les Allemands et les Néerlandais sont sensibles à l'existence } \\
\text { de similarités et de différences entre les VHA, VHB et VHC, } \\
\text { mais ils en connaissent moins au sujet de la transmission, des } \\
\text { conséquences et de la prévention de ces infections }\end{array}$ \\
\hline $\begin{array}{l}\text { Denniston et coll. } \\
(2012)(12) \\
\text { É.-U. }\end{array}$ & $\begin{array}{l}\text { Étude transversale } \\
\text { Population en général } \\
\text { qui a obtenu un test de } \\
\text { dépistage positif au VHC } \\
\text { ( } n=32847 \text { ) } \\
\text { Données NHANES } \\
\text { 2001-2008 }\end{array}$ & $\begin{array}{l}\text { Entrevue } \\
\text { téléphonique }\end{array}$ & $\begin{array}{l}\text { Conscience } \\
\text { - Moins de la moitié des personnes qui ont obtenu un test de } \\
\text { dépistage positif au VHC savaient qu'elles étaient infectées } \\
\text { Connaissances } \\
\text { - Les répondants ont répondu de manière correcte à la plupart des } \\
\text { questions de connaissance : on a constaté de } 57,1 \% \text { à } 95,7 \% \text { de } \\
\text { bonnes réponses } \\
\text { - Une plus faible proportion des répondants ont répondu } \\
\text { correctement aux questions liées à la transmission du VHC par } \\
\text { l'échange d'un baiser, les relations sexuelles et la transmission de } \\
\text { la mère à l'enfant } \\
\text { - Les réponses concernant la transmission verticale de la mère à } \\
\text { l'enfant ont reçu le plus de réponses «je ne sais pas» (33,7\%) }\end{array}$ \\
\hline
\end{tabular}


Appendice 1 : Description des études retenues $(n=19)$ (suite)

\begin{tabular}{|c|c|c|c|}
\hline $\begin{array}{l}\text { Auteurs(s), année } \\
\text { de publication et } \\
\text { pays }\end{array}$ & $\begin{array}{l}\text { Modèle de l'étude, } \\
\text { population et lieu }\end{array}$ & $\begin{array}{l}\text { Méthode de } \\
\text { la collecte de } \\
\text { données }\end{array}$ & Résultats et conclusions \\
\hline \multicolumn{4}{|c|}{ Population en général ( $n=14$ ) (suite) } \\
\hline $\begin{array}{l}\text { Eguchi et Wada (2013) } \\
(13) \\
\text { Japon }\end{array}$ & $\begin{array}{l}\text { Étude transversale } \\
\text { Population de travailleurs } \\
\text { japonnais ( } n=3129 \text { ) } \\
\text { En ligne }\end{array}$ & $\begin{array}{l}\text { Questionnaire } \\
\text { autoadministré } \\
\text { (version en ligne) }\end{array}$ & $\begin{array}{l}\text { Connaissances } \\
\text { - } 19 \% \text { croyaient que l'infection au VHB/VHC est la cause du cancer } \\
\text { du foie dans } 90 \% \text { des cas } \\
\text { - } 39 \% \text { croyaient que les personnes atteintes de VHB/VHC peuvent } \\
\text { contracter une cirrhose hépatique ou un cancer du foie à l'âge de } \\
40 \text { à } 60 \text { ans } \\
\text { - } 39 \% \text { croyaient que le traitement pouvait guérir les infections } \\
\text { VHB/VHC et prévenir les dommages hépatiques }\end{array}$ \\
\hline $\begin{array}{l}\text { Les Associés de } \\
\text { recherche EKOS inc. } \\
\text { (2012) (14) } \\
\text { Canada }\end{array}$ & $\begin{array}{l}\text { Étude transversale } \\
\text { Grand public ( } 16 \text { ans ou } \\
\text { plus) } \\
\text { ( } n=2000) \\
\text { Panel d'enquête }\end{array}$ & $\begin{array}{l}\text { Entrevue } \\
\text { téléphonique }\end{array}$ & $\begin{array}{l}\text { Conscience et connaissance } \\
\text { - La conscience n'était pas clairement définie } \\
\text { - } 13 \% \text { des Canadiens croyaient qu'ils connaissent très bien le VHC } \\
\text { - } 23 \% \text { croyaient que l'infection au VHC peut être guérie; } 55 \% \text { ne } \\
\text { le savaient pas ou n'ont pas donné de réponse } \\
\text { - } 50 \% \text { croyaient qu'un vaccin était disponible afin de prévenir le } \\
\text { VHC; } 24 \% \text { ne le savaient pas ou n'ont pas donné de réponse } \\
\text { - } 36 \% \text { ont indiqué que le VHC peut être transmis par les } \\
\text { transfusions sanguines } \\
\text { - } 25 \% \text { ont indiqué que le VHC peut être transmis par des relations } \\
\text { sexuelles non sécuritaires ou non protégées } \\
\text { - } 23 \% \text { ont indiqué que le VHC peut être transmis par la } \\
\text { consommation de drogues injectables et par l'échange d'aiguilles } \\
\text { - Moins de } 10 \% \text { ont indiqué que le VHC peut être transmis selon } \\
\text { les façons suivantes : simple contact (p. ex., échanger un baiser, } \\
\text { prendre quelqu'un dans ses bras, échanger une poignée de } \\
\text { main), de la mère à l'enfant au cours de la grossesse, en se faisant } \\
\text { tatouer ou en obtenant un perçage corporel, en échangeant des } \\
\text { articles d'hygiène corporelle } \\
\text { - } 25 \% \text { ne savaient pas ou n'avaient pas de réponse sur la façon } \\
\text { dont le VHC peut être transmis }\end{array}$ \\
\hline $\begin{array}{l}\text { Hopwood et coll. } \\
(2016)(15) \\
\text { Australie }\end{array}$ & $\begin{array}{l}\text { Étude transversale } \\
\text { Hommes homosexuels et } \\
\text { bisexuels atteints du VIH } \\
\text { ou du VHC }(n=474) \\
\text { Étude en ligne }\end{array}$ & $\begin{array}{l}\text { Questionnaire } \\
\text { autoadministré }\end{array}$ & $\begin{array}{l}\text { Connaissances } \\
\text { - La connaissance du VHC était de modérée à bonne } \\
\text { - } 44 \% \text { croyaient qu'être atteint du VIH augmentait la probabilité de } \\
\text { contracter le VHC au cours de relations sexuelles entre hommes } \\
\text { - Les répondants voulaient des renseignements sur la façon d'éviter } \\
\text { de transmettre le VHC à leurs partenaires sexuels (46\%), sur les } \\
\text { thérapies complémentaires au VHC (42\%), sur la manière dont } \\
\text { la co-infection au VIH/VHC affecte la santé (42\%) et sur la façon } \\
\text { dont les traitements du VIH et du VHC s'affectent l'un l'autre (40 } \\
\%) \\
\text { - La majorité des hommes ont mentionné que leur médecin } \\
\text { généraliste ou leur médecin spécialiste (85 \%) ou encore internet } \\
\text { (69\%) étaient leurs principales sources de renseignements sur le } \\
\text { VHC. Un nombre moins élevé d'hommes ont mentionné qu'ils } \\
\text { avaient obtenu des renseignements par des organismes en lien } \\
\text { avec l'hépatite (52\%), d'autres travailleurs du milieu de la santé } \\
\text { (38\%) et des amis (23\%) }\end{array}$ \\
\hline $\begin{array}{l}\text { Ipsos (2012) (16) } \\
\text { Canada }\end{array}$ & $\begin{array}{l}\text { Étude transversale } \\
\text { Population en général (18 } \\
\text { ans ou plus) ( } n=1000 \text { ) } \\
\text { (Milieu inconnu : } \\
\text { renseignement non } \\
\text { disponible) }\end{array}$ & & $\begin{array}{l}\text { Connaissances } \\
\text { - } 90 \% \text { ont mentionné qu'on peut être atteint de l'hépatite } C \text { sans } \\
\text { le savoir } \\
\text { - } 62 \% \text { de la génération Y ( } 18-29 \text { ans), } 60 \% \text { de la génération } X \\
\text { ( } 30-46 \text { ans) et } 54 \% \text { de la génération du baby-boom ( } 47-67 \text { ans) } \\
\text { savaient que le VHC était transmis principalement par le contact } \\
\text { sang à sang } \\
\text { - } 23 \% \text { de la génération Y ( } 18-29 \text { ans), } 14 \% \text { de la génération X } \\
\text { ( } 30-46 \text { ans) et } 18 \% \text { de la génération du baby-boom ( } 47-67 \text { ans) } \\
\text { savaient que l'infection au VHC était guérissable }\end{array}$ \\
\hline
\end{tabular}


Appendice 1 : Description des études retenues $(n=19)$ (suite)

\begin{tabular}{|c|c|c|c|}
\hline $\begin{array}{l}\text { Auteurs(s), année } \\
\text { de publication et } \\
\text { pays }\end{array}$ & $\begin{array}{l}\text { Modèle de l'étude, } \\
\text { population et lieu }\end{array}$ & $\begin{array}{l}\text { Méthode de } \\
\text { la collecte de } \\
\text { données }\end{array}$ & Résultats et conclusions \\
\hline \multicolumn{4}{|c|}{ Population en général ( $n=14$ ) (suite) } \\
\hline $\begin{array}{l}\text { Lambers et coll. (2013) } \\
\text { (17) } \\
\text { Pays-Bas }\end{array}$ & $\begin{array}{l}\text { Étude observationnelle } \\
\text { Hommes qui ont des } \\
\text { relations sexuelles avec } \\
\text { d'autres hommes, atteints } \\
\text { ou non du VIH } \\
\text { ( } \mathrm{n}=539) \\
\text { Variés (campagnes de } \\
\text { recrutement, média, } \\
\text { bouche-à-oreille) }\end{array}$ & $\begin{array}{l}\text { Questionnaire } \\
\text { autoadministré } \\
\text { (version papier)) }\end{array}$ & $\begin{array}{l}\text { Conscience } \\
\text { - } 74,1 \% \text { des répondants étaient sensibilisés au fait que le VHC } \\
\text { peut être transmis au cours de relations sexuelles entre hommes } \\
\text { et } 47,2 \% \text { au fait que les hommes porteurs du VIH sont plus } \\
\text { susceptibles de mentionner une transmission du VHC par voie } \\
\text { sexuelle } \\
\text { - } 57,5 \% \text { étaient sensibilisés au fait qu'il existe un traitement contre } \\
\text { le VHC et } 35,6 \% \text { au fait qu'il pouvait guérir l'infection } \\
\text { - } 23 \% \text { étaient sensibilisés au fait que le VHC pouvait disparaître } \\
\text { spontanément sans traitement } \\
\text { Connaissances } \\
\text { - Les participants avaient les meilleures notes sur les connaissances } \\
\text { relatives à la transmission du VHC et à la co-infection VIH/VHC } \\
\text { - Les participants avaient les plus basses notes sur la connaissance } \\
\text { relative à l'histoire naturelle du VHC, le dépistage et la prévention } \\
\text { ainsi que le traitement }\end{array}$ \\
\hline $\begin{array}{l}\text { Lea et coll. (2016) (18) } \\
\text { Australie }\end{array}$ & $\begin{array}{l}\text { Étude transversale } \\
\text { Hommes qui ont des } \\
\text { relations sexuelles avec } \\
\text { d'autres hommes ( } n=405 \text { ) } \\
\text { Variés (publicité sur les } \\
\text { réseaux sociaux, sites } \\
\text { Web d'organismes } \\
\text { communautaires) }\end{array}$ & $\begin{array}{l}\text { Questionnaire } \\
\text { autoadministré } \\
\text { (version en ligne) }\end{array}$ & $\begin{array}{l}\text { Conscience } \\
\text { - } 70 \% \text { des hommes qui ont des relations sexuelles avec d'autres } \\
\text { hommes sans être porteurs du VIH et } 80 \% \text { de ceux qui sont } \\
\text { porteurs du VIH étaient sensibilisés au VHC } \\
\text { - Plus de la moitié des hommes qui ont des relations sexuelles avec } \\
\text { d'autres hommes sans être porteurs du VIH (55\%) et ceux qui } \\
\text { sont porteurs du VIH ( } 63 \% \text { ) étaient sensibilisés à l'existence d'un } \\
\text { traitement contre le VHC } \\
\text { Connaissances } \\
\text { - } 31 \% \text { savaient que le VHC pouvait causer le cancer du foie } \\
\text { - } 37 \% \text { croyaient que le VHC pouvait provoquer une insuffisance } \\
\text { hépatique }\end{array}$ \\
\hline $\begin{array}{l}\text { Owiti et coll. (2015) (19) } \\
\text { Australie, Canada, } \\
\text { Mexique, Pays-Bas, } \\
\text { É.-U. }\end{array}$ & $\begin{array}{l}\text { Revue narrative } \\
\text { systématique } \\
\text { Prédominance } \\
{\text { d'immigrants } d^{\prime} \text { 'origine }}_{\text {asiatique }} \\
(\mathrm{n}=51)^{\mathrm{a}} \\
(\text { Milieu inconnu : } \\
\text { renseignement non } \\
\text { disponible) }\end{array}$ & $\begin{array}{l}\text { Renseignement non } \\
\text { disponible }\end{array}$ & $\begin{array}{l}\text { Connaissances } \\
\text { - De fausses idées étaient mentionnées concernant les différents } \\
\text { types d'hépatites (A, B, C) } \\
\text { - Des incertitudes sur l'histoire naturelle de l'hépatite (p. ex. les } \\
\text { dommages au foie) et de la confusion sur les causes (hormones, } \\
\text { stress) étaient présentes } \\
\text { - L'une des études examinées mentionnait le manque de } \\
\text { connaissance sur un traitement efficace contre le VHC } \\
\text { - On observait un faible niveau de connaissance sur les principaux } \\
\text { facteurs de risque de transmission, particulièrement sur les } \\
\text { contacts sexuels (transmission horizontale) et l'accouchement } \\
\text { (transmission verticale) } \\
\text { - La cause et la transmission étaient principalement attribuées, } \\
\text { de manière erronée, aux habitudes de vies et aux pratiques } \\
\text { culturelles liées à l'alimentation }\end{array}$ \\
\hline $\begin{array}{l}\text { Pundhir et coll. (2016) } \\
\text { (20) } \\
\text { É.-U. }\end{array}$ & $\begin{array}{l}\text { Étude transversale } \\
\text { Patients (18 ans ou plus) } \\
\text { atteints d'une infection au } \\
\text { VHC, avec ou sans } \\
\text { co-infection au VIH } \\
\text { ( } \mathrm{n}=292 \text { ) } \\
\text { Clinique de soins primaires }\end{array}$ & $\begin{array}{l}\text { Questionnaire } \\
\text { autoadministré } \\
\text { (versions papier et en } \\
\text { ligne) }\end{array}$ & $\begin{array}{l}\text { Connaissances } \\
\text { - Les répondants croyaient que, si leur médecin ne discutait pas de } \\
\text { I'hépatite C, il ne devait pas être important de la traiter } \\
\text { - Les répondants percevaient les longs temps d'attente avant de } \\
\text { consulter un médecin spécialiste pour un traitement comme un } \\
\text { signe qu'il n'était pas important de traiter la maladie } \\
\text { - La connaissance du VHC n'était pas associée à un comportement } \\
\text { d'assiduité aux rendez-vous }\end{array}$ \\
\hline
\end{tabular}


Appendice 1 : Description des études retenues $(n=19)$ (suite)

\begin{tabular}{|c|c|c|c|}
\hline $\begin{array}{c}\text { Auteurs(s), année } \\
\text { de publication et } \\
\text { pays }\end{array}$ & $\begin{array}{l}\text { Modèle de l'étude, } \\
\text { population et lieu }\end{array}$ & $\begin{array}{l}\text { Méthode de } \\
\text { la collecte de } \\
\text { données }\end{array}$ & Résultats et conclusions \\
\hline \multicolumn{4}{|c|}{ Population en général ( $n=14$ ) (suite) } \\
\hline $\begin{array}{l}\text { Rashrash et coll. (2016) } \\
\text { (21) } \\
\text { É.-U. }\end{array}$ & $\begin{array}{l}\text { Étude transversale } \\
\text { Afro-Américains de la } \\
\text { génération du baby-boom } \\
\text { (nés entre } 1945 \text { et 1965) } \\
\text { ( } n=137 \text { ) } \\
\text { Centres hospitaliers et de } \\
\text { mieux-être }\end{array}$ & $\begin{array}{l}\text { Enquête transversale } \\
\text { utilisant un système } \\
\text { d'auto-entrevues } \\
\text { audio assistées par } \\
\text { ordinateur }\end{array}$ & 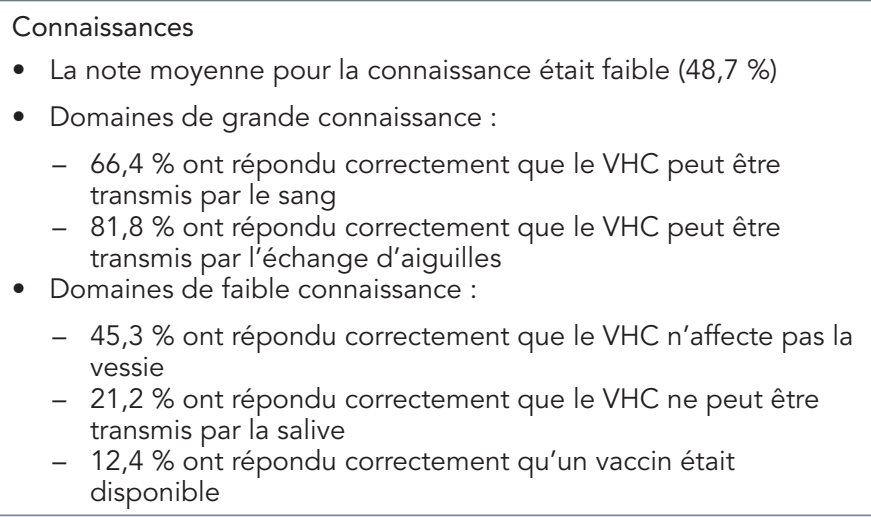 \\
\hline \multicolumn{4}{|c|}{ Fournisseurs de soins de santé $(n=6)$} \\
\hline $\begin{array}{l}\text { Bianco et coll. (2013) } \\
\text { (22) } \\
\text { Italie }\end{array}$ & $\begin{array}{l}\text { Étude transversale } \\
\text { Infirmiers }(n=326) \\
\text { Unités d'hémodialyse }\end{array}$ & $\begin{array}{l}\text { Questionnaire } \\
\text { autoadministré }\end{array}$ & $\begin{array}{l}\text { Connaissances } \\
\text { - } 49,8 \% \text { ont nommé correctement toutes les voies de transmission } \\
\text { du VHC } \\
\text { - La plupart des infirmiers ont nommé correctement certaines } \\
\text { des voies de transmissions parmi les suivantes : recevoir une } \\
\text { transfusion sanguine d'un donneur infecté }(93,9 \%) \text {, avoir des } \\
\text { relations sexuelles avec un partenaire porteur du VHC }(91,4 \%) \\
\text { et échanger des aiguilles lors de la consommation de drogues } \\
\text { injectables ( } 90,7 \%) \\
\text { - } 11,5 \% \text { croyaient que le VHC pouvait être transmis par l'échange } \\
\text { d'un baiser } \\
\text { - } 19,2 \% \text { n'ont pas mentionné que se faire tatouer était une voie de } \\
\text { transmission } \\
\text { - } 21,4 \% \text { croyaient de manière erronée qu'éviter l'allaitement } \\
\text { - } 70,8 \% \text { crouvait réduire les risques de transmission du VHC } \\
\text { de patient à patient }\end{array}$ \\
\hline $\begin{array}{l}\text { Ipsos (2012) (16) } \\
\text { Canada }\end{array}$ & $\begin{array}{l}\text { Étude transversale } \\
\text { Médecins généralistes et } \\
\text { médecins de famille } \\
\text { ( } \mathrm{n}=300 \text { ) } \\
\text { (Milieu inconnu: } \\
\text { renseignement non } \\
\text { disponible) }\end{array}$ & $\begin{array}{l}\text { Renseignement non } \\
\text { disponible }\end{array}$ & $\begin{array}{l}\text { Connaissances } \\
\text { - } 96 \% \text { des médecins généralistes croyaient que plusieurs } \\
\text { personnes porteuses du VHC n'ont pas connaissance de leur } \\
\text { infection } \\
\text { - } 35 \% \text { des médecins généralistes connaissent une foule de choses } \\
\text { sur les symptômes associés à l'infection au VHC } \\
\text { - } 10 \% \text { des médecins généralistes connaissent une foule de choses } \\
\text { sur les traitements disponibles } \\
\text { - } 43 \% \text { ont correctement répondu que l'hépatite C était } \\
\text { guérissable; } 22 \% \text { n'étaient pas certains }\end{array}$ \\
\hline $\begin{array}{l}\text { McGowan et coll. } \\
\text { (2013) (23) } \\
\text { Canada, Europe } \\
\text { centrale et Europe de } \\
\text { l'Est, Amérique latine, } \\
\text { Europe de l'Ouest, } \\
\text { pays nordiques, } \\
\text { pays asiatiques et du } \\
\text { Pacifique, Afrique et } \\
\text { Moyen-Orient, É.-U. }\end{array}$ & $\begin{array}{l}\text { Étude transversale } \\
\text { Médecins prodiguant le } \\
\text { traitement contre le VHC } \\
(\mathrm{n}=697) \\
\text { Base de données pour la } \\
\text { recherche sur le marché } \\
\text { international }\end{array}$ & $\begin{array}{l}\text { Entrevues } \\
\text { téléphoniques ou } \\
\text { questionnaires } \\
\text { autoadministrés }\end{array}$ & $\begin{array}{l}\text { Connaissances } \\
\text { - Globalement, une plus grande proportion d'hépatologues } \\
\text { connaissaient le traitement contre le VHC comparativement aux } \\
\text { médecins généralistes } \\
\text { - La plupart des médecins comprenaient que différents génotypes } \\
\text { nécessitent différentes durées de traitement } \\
\text { - La plupart des médecins comprenaient que le traitement doit être } \\
\text { interrompu chez les patients sans réponse virologique précoce } \\
\text { - La majorité des médecins croyaient de manière erronée que les } \\
\text { niveaux d'ARN associés au VHC étaient corrélés avec la gravité de } \\
\text { la maladie hépatique } \\
\text { - La majorité des médecins croyaient aussi à tort que les patients } \\
\text { qui ne répondent pas au traitement devraient subir une thérapie } \\
\text { d'entretien } \\
40 \% \text { des fournisseurs croyaient qu'ils possédaient une } \\
\text { connaissance adéquate des lignes directrices du traitement }\end{array}$ \\
\hline
\end{tabular}


Appendice 1 : Description des études retenues $(n=19)$ (suite)

\begin{tabular}{|c|c|c|c|}
\hline $\begin{array}{l}\text { Auteurs(s), année } \\
\text { de publication et } \\
\text { pays }\end{array}$ & $\begin{array}{l}\text { Modèle de l'étude, } \\
\text { population et lieu }\end{array}$ & $\begin{array}{l}\text { Méthode de } \\
\text { la collecte de } \\
\text { données }\end{array}$ & Résultats et conclusions \\
\hline \multicolumn{4}{|c|}{ Fournisseurs de soins de santé ( $n=6$ ) (suite) } \\
\hline $\begin{array}{l}\text { Naghdi et coll. (2017) } \\
\text { (24) } \\
\text { Canada }\end{array}$ & $\begin{array}{l}\text { Étude transversale } \\
\text { Médecins de première } \\
\text { ligne, médecins } \\
\text { spécialistes, infirmiers en } \\
\text { hépatologie et infirmiers } \\
\text { praticiens ( } \mathrm{n}=163 \text { ) } \\
\text { Échantillon de commodité } \\
\text { par les organismes des } \\
\text { fournisseurs }\end{array}$ & $\begin{array}{l}\text { Questionnaire } \\
\text { autoadministré } \\
\text { (version en ligne) }\end{array}$ & $\begin{array}{l}\text { Connaissances } \\
\text { - } 78 \% \text { des médecins de première ligne éprouvaient un malaise à } \\
\text { faire commencer la thérapie contre l'hépatite C } \\
\text { - } 70 \% \text { des médecins de première ligne ont exprimé leur malaise à } \\
\text { substituer une thérapie pour une autre face à leurs patients } \\
\text { - Comparativement aux médecins de première ligne, les } \\
\text { hépatologues, les gastroentérologues, les infirmiers en } \\
\text { hépatologie et les infirmiers praticiens ont exprimé une plus } \\
\text { grande aisance à assurer le suivi de la thérapie actuelle des } \\
\text { patients } \\
\text { - } 22 \% \text { des médecins de première ligne étaient peu sensibilisés à } \\
\text { l'état actuel de la thérapie contre le VHC }\end{array}$ \\
\hline $\begin{array}{l}\text { Rotte et coll. (2013) (25) } \\
\text { É.-U. }\end{array}$ & $\begin{array}{l}\text { Étude observationnelle } \\
\text { Résidents, médecins, } \\
\text { infirmiers praticiens, } \\
\text { adjoints au médecin } \\
\text { ( } \mathrm{n}=78 \text { ) } \\
\text { Services des urgences }\end{array}$ & $\begin{array}{l}\text { Questionnaire } \\
\text { autoadministré } \\
\text { (version en ligne) }\end{array}$ & $\begin{array}{l}\text { Connaissances } \\
\text { - La connaissance des conséquences du VHC était élevée } \\
\text { (pourcentage non disponible) } \\
\text { - } 81 \% \text { n'étaient pas sensibilisés au fait que des médicaments } \\
\text { pouvant guérir l'infection au VHC étaient disponibles } \\
\text { - } 58 \% \text { étaient sensibilisés aux lignes directrices relatives au VHC du } \\
\text { Centre pour le contrôle et la prévention des maladies américains } \\
\text { (CDC) } \\
\text { - } 42 \% \text { éprouvaient des inquiétudes face à la possibilité de } \\
\text { contracter le VHC dans le cadre de leur travail au service des } \\
\text { - } 67 \% \text { épres } \\
\text { - } 71 \% \text { convaient plus d'inquiétude de contracter le VHC en se } \\
\text { bénéfique pourent quíun deurs dépistage précoce du VHC serait } \\
\text { - } 40 \% \text { ne croyaient pas que des fournisseurs de soins de santé } \\
\text { atteints du VHC pouvaient transmettre l'infection à un patient }\end{array}$ \\
\hline $\begin{array}{l}\text { Todorova et coll. (2015) } \\
\text { (26) } \\
\text { Bulgarie }\end{array}$ & $\begin{array}{l}\text { Étude transversale } \\
\text { Étudiants en art dentaire } \\
(\mathrm{n}=96) \\
\text { Faculté de médecine } \\
\text { dentaire, Université } \\
\text { médicale de Varna, } \\
\text { Bulgarie }\end{array}$ & $\begin{array}{l}\text { Questionnaire } \\
\text { autoadministré }\end{array}$ & $\begin{array}{l}\text { Connaissances } \\
\text { - } 41,6 \% \text { présentaient une bonne connaissance du VHB/VHC (note } \\
\text { de } 8 / 10) \\
\text { - Sensibilisés aux possibles voies de transmission : } \\
\text { - } 90,6 \% \text { connaissaient les lésions à la peau ou la transmission } \\
\text { par le sang } \\
\text { - } 62,5 \% \text { connaissaient les lésions à la peau ou la salive } \\
\text { - } 87,5 \% \text { connaissaient les blessures avec aiguilles } \\
\text { - Le contact peau intacte et salive }(87,5 \% \text { ) et le contact peau } \\
\text { intacte à peau intacte ( } 90,6 \% \text { ) étaient considérées de façon } \\
\text { correcte comme étant non dangereux pour la transmission du } \\
\text { VHB/VHC et respectivement } \\
\text { - } 80 \% \text { savaient que les porteurs du VHB/VHC pouvaient avoir l'air } \\
\text { en santé et ne pas présenter de symptômes apparents }\end{array}$ \\
\hline
\end{tabular}

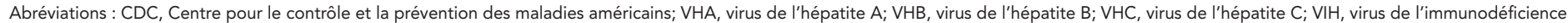
humaine; NHANES, National Health and Nutrition Examination Survey; ARN, acide ribonucléique

a Un certain nombre d'études ont été intégrées dans l'examen systématique 